\title{
Posttraumatic Growth of Adolescents with Childhood Leukemia and their Parents
}

\author{
Sungsil Hong ${ }^{1}$, Ho Ran Park ${ }^{2}$, Sun Hee Choi ${ }^{3}$ \\ ${ }^{1}$ Assistant Professor, Department of Nursing, College of Health Science, Kangwon National University, Samcheok; ${ }^{2}$ Professor, College of Nursing, The Catholic \\ University of Korea, Seoul; ${ }^{3}$ Unit Manager, Seoul St. Mary's Hospital, The Catholic University of Korea, Seoul, Korea
}

Purpose: Childhood leukemia is a serious trauma affecting both adolescents and their parents, who experience painful process. However, adolescents with leukemia and their parents also experience positive changes, which is referred to as posttraumatic growth. We examined posttraumatic growth, core beliefs, impact of event, and event-related rumination in adolescents within 5 years of a diagnosis of childhood leukemia and their parents. Methods: The participants were 68 adolescents with childhood leukemia (aged 13 18 years) and their parents, who were recruited from C university hospital in Korea from May to September 2016. The Posttraumatic Growth Inventory, Core Belief Inventory, Impact of Event Scale-Revised, and Event-related Rumination Inventory were completed by the adolescents and their parents. The mean scores and correlations between variables were investigated for both set of participants. Results: Parents showed significantly higher levels of posttraumatic growth, disruption of core beliefs, impact of event, and invasive rumination than adolescents. Disruption of core beliefs and deliberate rumination were positively correlated with posttraumatic growth in both groups. Conclusion: Nursing intervention programs that involve modifying core beliefs and inducing a positive thought can help adolescents with leukemia and their parents grow after traumatic events.

Key words: Posttraumatic stress disorder; Adolescents; Parents; Acute lymphoblastic leukemia

\section{Corresponding author Sungsil Hong \\ https://orcid.org/0000-0002-4346-322X}

Department of Nursing, College of Health Science, Kangwon National University, 346 Hwangjo-gil, Dogye-eup, Samcheok 25949, Korea

TEL +82-33-540-3366 FAX +82-33-540-3369

E-MAIL hss9456@gmail.com

*This work was supported by a 2015 Research Grant from Kangwon National University, Korea.

Received Jul 17, 2018 Revised Aug 21, 2018 Accepted Sep 3, 2018
(2) This is an Open Access article distributed under the terms of the Creative Commons Attribution NonCommercial License (http://creativecommons.org/licenses/by-nc/4.0/) which permits unrestricted noncommercial use, distribution, and reproduction in any medium, provided the original work is properly cited.

\section{INTRODUCTION}

A diagnosis of childhood cancer is a traumatic experience not only for adolescents, who are directly affected by the illness, but also for their parents, who are present at every step of the arduous journey to recovery. A previous study reported that over $50 \%$ of children with cancer remember the diagnosis and treatment process as a traumatic event, resulting in post-traumatic stress [1]. In contrast, another study reported that $85 \%$ of childhood cancer survivors identified at least one positive consequence from the challenging experience [2], suggesting that the negative experience can be transcended by positive effects.

Posttraumatic growth (PTG) is defined as the "positive psychological change experienced as a result of the struggle with highly challenging life circumstances." [3]. In the PTG model developed by Calhoun and Tedeschi [4], emotional shock by a seismic event, disruption of core beliefs, and rumination as cognitive processing are proposed as vital themes leading to PTG. In other words, a traumatic event shakes an individual's fundamental schema, beliefs, and goals, causing severe stress. This is followed by rumination as a way of cognitive processing. In the beginning, intrusive rumination prevails, independent of the individual's will, which can intensify the level 
of stress he or she experiences. In time, however, the individual engages in deliberate rumination, where he or she repeatedly contemplates the positive legacy of the event. This process leads to schema change, development of a new outlook regarding life's challenges, and eventual PTG (Figure 1).

Naturally, stressful circumstances due to a severe illness such as cancer are often detrimental to adolescents' physical and emotional development, and this can have a lasting negative impact on their re-adjustment to society following treatment and recovery [5]. Before adolescence, children find it hard to understand their illness clearly and cannot express their thinking and/or opinions about the disease and treatments; moreover, they passively follow the treatment process according to their parents' decisions. However, adolescence is a period when self-identity and autonomy are established, during which adolescents' relationship with their parents changes from dependent to interdependent. Adolescents take initiative and make decisions on their own, rather than following their parents' decisions [6]. Adolescents with childhood leukemia experience repeated adjustment and maladjustment in a crisis situation of the diagnosis and treatment of disease, and as a result, they grow up as mature being who can transform the meaning of disease into a positive experi- ence [7]. When adolescents have a life-threatening disease, such as childhood cancer, they actively participate in treatment, especially if they are willing to cope with the disease. Although parents spend considerable amounts of time with their children, which may be emotionally painful, they tend to focus on helping their children. Sometimes parents spend large amounts of time with their children, without expressing pain and suffering to them. In family-oriented Korean culture, the relationship between children and parents is more intense than in Western culture, and many parents tend to make most decisions for their children until they become adults. In patients with childhood cancers, the parents of the affected children typically go through the traumatic experience of diagnosis and treatment alongside their child. As such, parents' feelings about the traumatic event and their coping play a meaningful role in the child's experience of the event [8]. Supporting affected adolescents and parents in coping with the trauma related to cancer diagnosis and treatment and helping them successfully adapt to changes may support them as they develop the ability to function as viable members of society.

Adolescents' traumatic experiences of childhood leukemia are expected to differ from the traumatic experiences of their parents as caregivers or guardians $[2,9,10]$. These differences

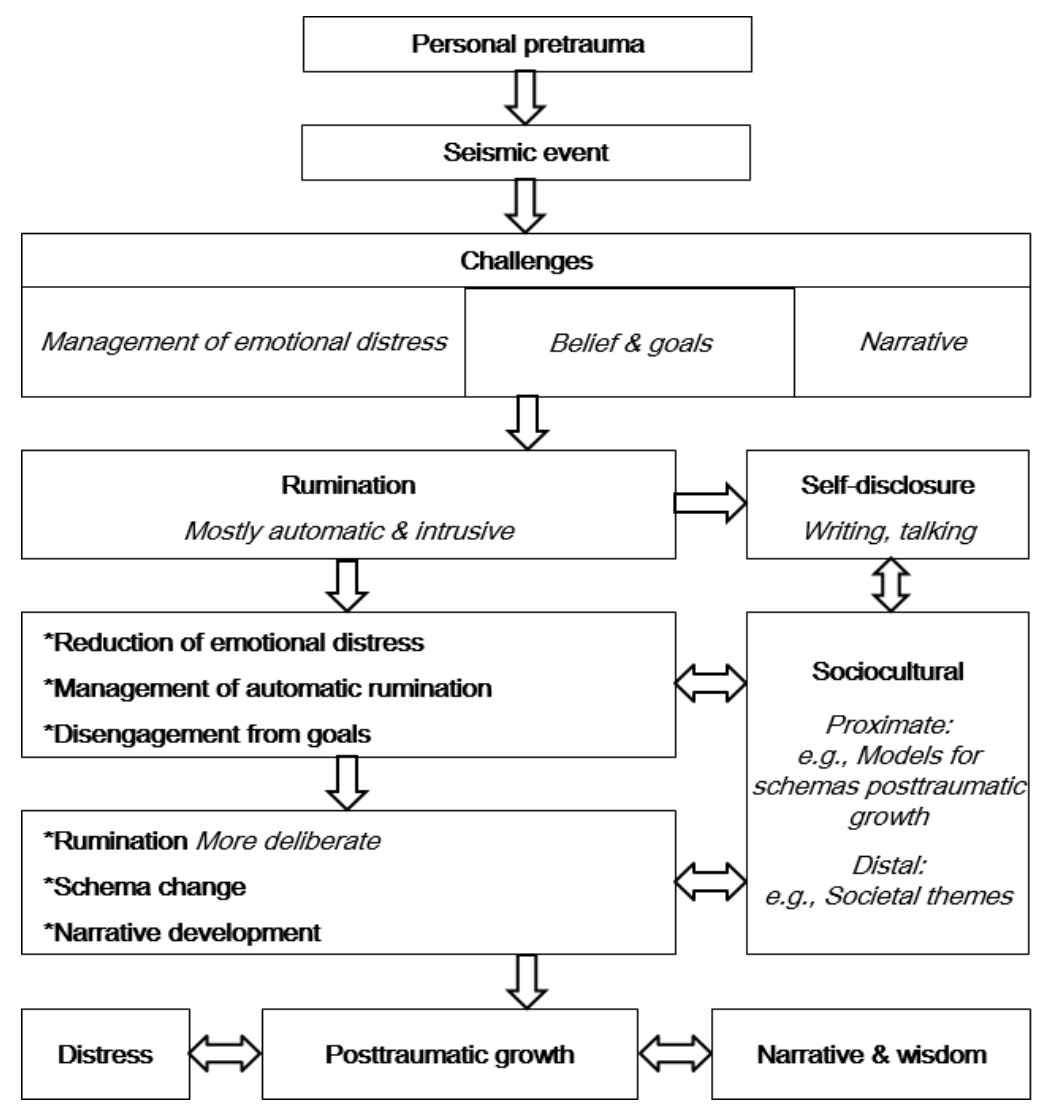

Figure 1. The theoretical model of posttraumatic growth presented by Calhoun and Tedeschi (2006). 
in perspective should be separately considered in the assessment, planning, and implementation of nursing interventions for adolescents and their parents. According to the PTG model by Calhoun and Tedeschi [4], core belief, level of impact, intrusive rumination, and deliberate rumination are core factors that determine PTG, even though PTG can also be affected by various individual and psychosocial variables. Until now, most papers have studied PTG in adolescents with childhood cancer and their parents separately. Only a few previous studies have investigated this topic among both adolescents with cancer and their parents [2,11], and those studies did not explicitly include PTG and the vital themes based on the PTG model.

Therefore, we examined PTG in adolescents with childhood leukemia, the most common childhood cancer in Korea, and their parents to identify the relationships between the vital themes and PTG that were proposed in the PTG model by Calhoun and Tedeschi [4]. We aimed to further understand the level of PTG and its dynamics to develop a tailored nursing intervention program that promotes PTG in Korean adolescents with childhood leukemia and their parents.

\section{METHODS}

\section{Study Design}

This study was a descriptive cross-sectional survey conducted to identify PTG and core themes perceived by adolescents with childhood leukemia and their parents.

\section{Participants and Procedures}

The participants were 68 adolescents with childhood leukemia and their parents, who were recruited from $C$ university hospital in Korea from May to September 2016. The adolescent participants satisfied the following selection criteria: 1) having been diagnosed with childhood leukemia within 5 years, 2) having completed consolidation therapy and currently receiving outpatient care, 3 ) being aged 13-18 years, and 4) being capable of comprehending the survey questionnaire and communicating with the researcher. The parent participants satisfied the following selection criteria: 1) being a parent of a child with childhood leukemia and 2) being capable of comprehending the survey questionnaire and communicating with the researcher. The required sample size for this study was estimated using G*Power 3.1.5 [12]. When input into a paired t-test analysis using $\alpha=.05$, a medium effect size of $d=.50$, and power $=.95$, the minimum sample size for this study was 54 for each group. Considering the potential dropout rate, 68 per group were used as sample sizes in this study.
Researcher explained the purpose of the study to the adolescents and their parents at the outpatient clinic. While the adolescent and parent completed the informed consent form and filled out the questionnaire, a researcher stayed with them in order to reply to any possible questions. It took roughly $30 \mathrm{mi}-$ nutes to complete the questionnaire, and participants were compensated with a souvenir (worth 10 US dollars) upon completion of the questionnaire.

\section{Ethical Considerations}

We obtained the approval of C University Hospital's Institutional Review Board (No. **16QISI0272) and benefited from the cooperation of attending physicians and relevant departments. The researcher explained the purpose of the study and the content of the questionnaire to the adolescents and parents who met the criteria. Those who agreed to participate in the study completed a written consent form and filled out a questionnaire.

\section{Instruments}

The original authors of all the instruments utilized in this study approved their use, and verified Korean versions of all instruments were administered. In order to apply the instruments to adolescents, we asked 3 adolescents with childhood leukemia and their parents to check the wording in the questionnaire and to provide feedback. We started the study after modifying certain words and expressions to be more appropriate for adolescents.

\section{1) Evaluation of PTG and its variables}

(1) Demographic characteristics and disease-related characteristics

Adolescents' and parents' age at the time of the study, sex, religion, age at diagnosis, type of leukemia, and treatment type were collected.

\section{(2) PTG Inventory (PTGI)}

We used the validated Korean adaptation of Tedeschi and Calhoun's [13] PTGI (K-PTGI) [14] to measure the perceived positive legacy following a traumatic experience. The K-PTGI included 21 items, and each question was scored on a 6-point Likert scale (0: did not experience change to 5: experienced this change to a very great degree), with higher scores indicating a greater level of perceived positive legacy. The scale's reliability is acceptable: Cronbach's $\alpha=.90$ in Tedeschi and Calhoun's [13] study, Cronbach's $\alpha=.91$ in the study of Song et al. [14], and Cronbach's $\alpha=.91$ and .94 for adolescents and parents, respectively, in this study. 
(3) Core Belief Inventory (CBI)

We used the Korean version of the CBI, adapted by Jo [15] from the original instrument developed by Cann et al. [16]. Disruption of core beliefs refers to the degree that an individual reviews his or her core beliefs as they pertain to the self and the world after a traumatic event [15]. Higher scores indicate a greater breakdown of core beliefs. The scale consists of 9 items, each scored on a 5-point Likert scale (1: not at all true to 5: very true). The instrument's reliability is acceptable: Cronbach's $\alpha=.82$ in the study of Cann et al. [16], Cronbach's $\alpha=.81$ in Jo's study [15], and Cronbach's $\alpha=.83$ and .87 for adolescents and parents, respectively, in this study.

\section{(4) Impact of Event Scale-Revised (IES-R)}

To measure the impact of the leukemia diagnosis, the IES-R, which was developed by Weiss and Marmar [17] and translated into Korean [18], was used. This self-reporting scale consists of 22 items across 3 domains: intrusion, avoidance, and hyperarousal. Each item is scored on a 5-point Likert scale (0: not at all to 4: extremely). A higher total score indicates a greater level of distress associated with a traumatic event. The instrument's reliability is acceptable: Cronbach's $\alpha=.89 \sim .94$ in the original study [17], Cronbach's $\alpha=.93$ in the study of Lim et al. [18], and Cronbach's $\alpha=.94$ and .94 for adolescents and parents, respectively, in this study.

(5) K-Event Related Rumination Inventory (K-ERRI)

To measure the degree of rumination an individual engages following a traumatic event, we used the K-ERRI [19], which is a validated Korean adaptation of the ERRI developed by Cann et al. [20]. The K-ERRI consists of 20 items: 10 items corresponding to intrusive rumination, which is the type of thinking that repeatedly occurs against an individual's will following a traumatic experience; and 10 items corresponding to deliberate rumination, which is the type of thinking the individual repeatedly and intentionally engages in as a way of making sense of the event [20]. Each item is scored on a 4point Likert scale (0: never to 3: often). Higher scores indicate a greater level of the corresponding rumination type. The instrument's reliability is acceptable: Cronbach's $\alpha=.80 \sim .93$ in the original study [20], Cronbach's $\alpha=.95 \sim .96$ for the K-ERRI [19] and Cronbach's $\alpha=.90 \sim .93$ and .94 .95 for adolescents and parents, respectively, in this study.

\section{Statistical Analysis}

The collected data were analyzed using SPSS/WIN 19.0. Participants' demographic characteristics, disease-related characteristics, and the level of posttraumatic growth, disruption in core beliefs, impact of event, and intrusive/deliberate ru- mination were presented as numeric measurements, percentages, means, and standard deviations. PTG according to participants' demographic and disease-related characteristics was analyzed with the t-test and analysis of variance. To compare the PTG variables between groups, the paired t-test and the Wilcoxon signed rank test were performed. The Pearson correlation coefficient was used to examine the relationships between the variables.

\section{RESULTS}

\section{Demographic and Disease-related Characteristics and PTG according to Participants' Characteristics}

Participants' demographic and disease-related characteristics are displayed in Table 1.

Participants' PTG scores are shown in Table 1. Among adolescents, those with a religious affiliation showed a significantly higher level of PTG relative to those without $(\mathrm{t}=2.29$, $p=.025$ ). Mothers showed a significantly higher level of PTG than did fathers $(\mathrm{t}=2.09, p=.040)$ and parents with a religious affiliation showed a significantly higher level of PTG than did those without $(\mathrm{t}=3.58, p=.001)$. There were no differences in adolescents' and parents' PTG scores according to other general characteristics.

\section{Differences in Study Variables between Adolescents and Parents}

The mean scores for PTG, disruption of core beliefs, impact of events, and intrusive rumination differed significantly between adolescents and parents; however, there was no difference in deliberate rumination. Across all variables, the parents showed higher mean scores than the adolescents (Figure 2).

\section{Correlations between Participants' PTG, Disruption of Core Beliefs, Impact of Event, and Event-related}

\section{1) Rumination}

PTG was positively correlated with disruption of core beliefs and deliberate rumination in both adolescents and parents (Table 2). In other words, the higher the level of core belief collapse, the higher the level of deliberate rumination, the higher the PTG level. However, impact of event and intrusive rumination were not correlated with PTG in either group.

\section{DISCUSSION}

Diagnosis of leukemia is a traumatic event causing severe distress in affected adolescents and their parents. Although 
Table 1. Posttraumatic Growth according to Participants' Characteristics

$(N=136)$

\begin{tabular}{|c|c|c|c|c|c|c|}
\hline \multirow{2}{*}{ Variables } & \multirow{2}{*}{ Characteristics } & \multirow{2}{*}{ Categories } & \multirow{2}{*}{$\begin{array}{c}\mathrm{n}(\%) \text { or } \\
\mathrm{M} \pm \mathrm{SD}\end{array}$} & \multirow{2}{*}{ Range } & \multicolumn{2}{|c|}{ Posttraumatic growth } \\
\hline & & & & & $\mathrm{M} \pm \mathrm{SD}$ & t or $F(p)$ \\
\hline \multirow[t]{8}{*}{ Adolescents $(\mathrm{n}=68)$} & & & & & $71.22 \pm 15.63$ & \\
\hline & Age at study (year) & & $16.09 \pm 1.73$ & $13 \sim 18$ & & \\
\hline & Age at diagnosis (year) & & $13.65 \pm 2.18$ & $9 \sim 18$ & & \\
\hline & Gender & $\begin{array}{l}\text { Male } \\
\text { Female }\end{array}$ & $\begin{array}{l}41(60.3) \\
27(39.7)\end{array}$ & & $\begin{array}{l}70.12 \pm 15.72 \\
72.89 \pm 15.64\end{array}$ & $0.71(.479)$ \\
\hline & Religion & $\begin{array}{l}\text { Yes } \\
\text { None }\end{array}$ & $\begin{array}{l}35(51.5) \\
33(48.5)\end{array}$ & & $\begin{array}{l}75.31 \pm 11.77 \\
66.88 \pm 18.08\end{array}$ & $2.29(.025)$ \\
\hline & Diagnosis & $\begin{array}{l}\text { Acute lymphoblastic leukemia } \\
\text { Acute myeloblastic leukemia } \\
\text { Chronic myeloblastic leukemia }\end{array}$ & $\begin{array}{c}44(64.7) \\
21(30.9) \\
3(4.4)\end{array}$ & & $\begin{array}{l}71.32 \pm 15.80 \\
72.29 \pm 15.12 \\
62.33 \pm 20.11\end{array}$ & $0.53(.593)$ \\
\hline & Treatment type & $\begin{array}{l}\text { Chemotherapy only } \\
\text { Chemotherapy and transplantation } \\
\text { Chemotherapy, transplantation, } \\
\text { and radiation therapy }\end{array}$ & $\begin{array}{r}45(66.2) \\
14(20.6) \\
9(13.2)\end{array}$ & & $\begin{array}{l}71.69 \pm 17.22 \\
68.14 \pm 11.51 \\
73.67 \pm 13.30\end{array}$ & $0.39(.676)$ \\
\hline & $\begin{array}{l}\text { Treatment } \\
\text { completion }\end{array}$ & $\begin{array}{l}\text { Yes } \\
\text { No }\end{array}$ & $\begin{array}{l}28(41.2) \\
40(58.8)\end{array}$ & & $\begin{array}{l}74.07 \pm 15.08 \\
69.23 \pm 15.89\end{array}$ & $1.26(.211)$ \\
\hline \multirow[t]{4}{*}{ Parents $(n=68)$} & & & & & $79.12 \pm 16.12$ & \\
\hline & Age (year) & & $46.26 \pm 4.00$ & $38 \sim 55$ & & \\
\hline & Relation & $\begin{array}{l}\text { Father } \\
\text { Mother }\end{array}$ & $\begin{array}{l}10(14.7) \\
58(85.3)\end{array}$ & & $\begin{array}{l}69.50 \pm 13.82 \\
80.76 \pm 16.01\end{array}$ & $2.09(.040)$ \\
\hline & Religion & $\begin{array}{l}\text { Yes } \\
\text { None }\end{array}$ & $\begin{array}{l}46(67.6) \\
22(32.4)\end{array}$ & & $\begin{array}{l}83.57 \pm 13.19 \\
69.77 \pm 17.94\end{array}$ & $3.58(.001)$ \\
\hline
\end{tabular}

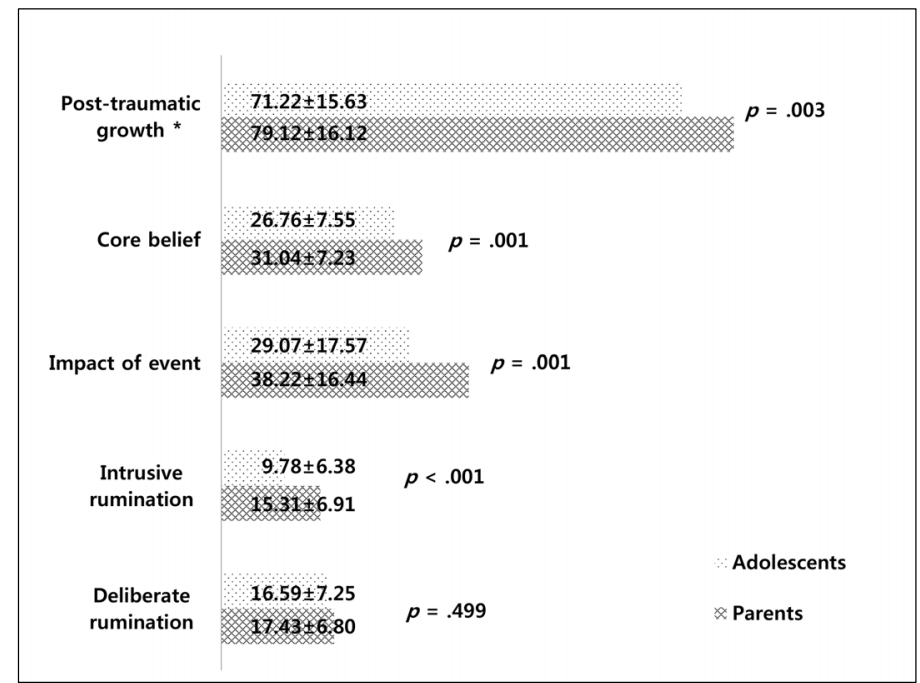

Figure 2. Differences in study variables between adolescents and parents ( $M \pm S D)$.

there is growing interest in the PTG of childhood cancer survivors and their families [2,11], the correlations of PTG with related variables in adolescents with childhood leukemia and their parents, such as those based on the core concepts of the PTG model, have rarely been studied. We characterized the relationships between adolescents' and their parents' PTG and relevant variables.

The mean PTGI scores among adolescents and parents were higher than those measured with the same scale in previous studies involving childhood cancer survivors [9] or 
Table 2. Correlations between Participants' Posttraumatic Growth and Vital Themes

\begin{tabular}{|c|c|c|c|c|c|c|}
\hline \multirow[t]{2}{*}{ Variables } & \multirow[t]{2}{*}{ Categories } & $\begin{array}{c}\text { Posttraumatic } \\
\text { growth }\end{array}$ & Core belief & $\begin{array}{c}\text { Impact of } \\
\text { event }\end{array}$ & Intrusive & Deliberate \\
\hline & & $\mathrm{r}(p)$ & $\mathrm{r}(p)$ & $\mathrm{r}(p)$ & $\mathrm{r}(p)$ & $\mathrm{r}(p)$ \\
\hline \multirow{5}{*}{ Adolescents } & Posttraumatic growth & 1 & $.61(<.001)$ & $-.05(.669)$ & $-.08(.536)$ & $.43(<.001)$ \\
\hline & Core belief & & 1 & $.13(.299)$ & $.01(.921)$ & $.52(<.001)$ \\
\hline & Impact of event & & & 1 & $.78(<.001)$ & $.14(.259)$ \\
\hline & Event-related intrusive rumination & & & & 1 & $.12(.343)$ \\
\hline & Event-related deliberate rumination & & & & & 1 \\
\hline \multirow[t]{5}{*}{ Parents } & Posttraumatic growth & 1 & $.77(<.001)$ & $.10(.407)$ & $-.01(.916)$ & $.32(.008)$ \\
\hline & Core belief & & 1 & $.22(.067)$ & $.01(.909)$ & $.28(.020)$ \\
\hline & Impact of event & & & 1 & $.60(<.001)$ & $.25(.040)$ \\
\hline & Event-related intrusive rumination & & & & 1 & $.57(<.001)$ \\
\hline & Event-related deliberate rumination & & & & & 1 \\
\hline
\end{tabular}

adult patients [21]. The mean PTGI score among the parents in this study was higher than those found among the parents of children with chronic illnesses or childhood cancer in previous studies [8,22]. Our results indicated that adolescents with childhood leukemia and their parents had moved beyond the despair and distress caused by the diagnosis to discover new meanings and beliefs, a process that ultimately led to PTG. The elevated levels of PTG among the study's participants may be attributed to their entry into a relatively stable period in their treatment journey, since consolidation therapy had just been completed and the severe distress and anxiety directly following the leukemia diagnosis had subsided. A reason why they showed positive scores may have been that the 5-year survival rate for childhood leukemia in Korea has increased to approximately $80 \%$ because of recent advances in medical technology [23]. For adolescents with childhood leukemia and their parents, leukemia was no longer an incurable disease, but a kind of short-term nightmare. A previous study reported that survivors of childhood cancer experienced a more abundant and satisfying life after suffering a life-threatening disease [24]. PTG is a dynamic concept in which various related factors emerge through mutual interactions [4]. Therefore, a qualitative and/or hybrid method will help to understand the concept of trauma in adolescents and parents and to obtain a deeper understanding of the process of overcoming and maturation.

A comparison of the PTG scores between fathers and mothers showed that mothers achieved a significantly higher level of PTG. Prior findings regarding this have been inconsistent, with some reporting a higher level of PTG in mothers than in fathers $[22,25]$. The reason why mothers showed a higher score than fathers may stem from the differences in coping strategies in traumatic situations between women and men. In general, women use more emotion-focused coping strategies [3], and are more likely to use strategies including verbal expression to seek emotional support [26]. In Korean culture, mothers take care of sick children as the primary caregivers and play a key role in providing emotional support for their children. Additionally, mothers are more often present during diagnosis and treatment and actively participate in medical decision-making. These factors seem to contribute to the tendency for mothers to experience higher levels of PTG than fathers.

The higher levels of PTG among religious adolescents and parents relative to their non-religious counterparts agree with the findings of previous studies $[8,13]$. Individuals in stressful circumstances tend to seek solace in an absolute power, and religious faith may provide the strength required to overcome specific challenges [8]. In this sense, strengthening faith may help adolescents with leukemia and their parents achieve PTG.

We found no significant difference in PTG levels by demographic characteristics, except for parent's sex and religious practices. This is probably because the inclusion criteria were limited to adolescents with childhood leukemia diagnosed within 5 years and their parents; therefore, the results did not reflect major group differences. According to the PTG model by Calhoun and Tedeschi [4], PTG is influenced by various psychosocial factors in addition to those examined in this study, suggesting that a longitudinal follow-up study that incorporates a more diverse set of variables would be useful.

Higher levels of PTG, disruption of core beliefs, impact of event, and intrusive rumination were found among parents than among adolescents. Although a direct comparison is not possible because of the lack of data collected from the same set of participants using the same scales, the parents experienced more disruption in core beliefs than their children, which was 
linked to a greater level of PTG. A notable finding is that the mean impact of event scores among adolescents and parents were higher than those found in previous studies dealing with osteosarcoma [11]. This may have been due to differences in diagnosis, the treatment process, and prognosis. According to the PTG model [4], positive changes from posttraumatic stress that lead to growth start with the disruption of core beliefs. The greater disruption of core beliefs among parents is the first factor contributing to higher levels of PTG. Parents generally gain wisdom and lessons from their lives to a greater extent than adolescents, and may have better knowledge of how to use sources of support. Parents who have achieved PTG can also be a major sources of support for their child.

PTG in adolescents and parents showed significant positive correlations with disruption of core beliefs and deliberate rumination, supporting the findings of previous studies [4,22]. Continuous efforts by an individual to restructure preexisting beliefs and faith in a stressful situation, and to view the challenges under a positive light, were confirmed to be crucial factors contributing to PTG. However, we did not find a correlation between PTG and impact of event, which represents the level of distress associated with the event. Although this finding is consistent with a previous study concerning oncology patients [27], it is inconsistent with that of another study concerning parents [11]. Because PTG is a dynamic concept influenced by multiple variables, a future longitudinal study that tracks the changes in variables among adolescents and their parents from the point of diagnosis would be beneficial.

This study has some limitations. Because we recruited participants from a single hospital, the results may not be generalizable to all adolescents and parents affected by childhood leukemia or childhood cancers. Furthermore, because this was a cross-sectional study, we are limited in our ability to infer causation or to assess PTG changes over time. In a follow-up study, the analysis will be expanded to include location and type of cancer in participants, and a longitudinal design that incorporates a more diverse set of variables will be implemented. Another limitation was that we did not collect enough data concerning participants' family situation, such as family type and economic status. Further studies need to consider more demographic factors related to PTG.

\section{CONCLUSION}

Life-threatening diseases such as leukemia have a negative emotional impact on affected adolescents and their parents. However, they can provide an opportunity for progress and growth. In this study, the parents of affected adolescents showed significantly higher levels of PTG, disruption of core beliefs, impact of event, and intrusive rumination than the adolescents. In addition, in both adolescents and parents, the more their core beliefs were shaken, the more readily they could view the stressful situation under a positive light. Disruption of core beliefs also made participants more likely to engage in deliberate rumination and was associated with higher PTG levels. This study is expected to improve our understanding of PTG among adolescents with childhood leukemia and parents, which is important given increases in the survival rate, and it is significant that this study focused on PTG with vital themes based on the PTG model. These results suggest that, to foster PTG in adolescents with leukemia and their parents, nursing interventions designed to help the affected adolescents and their parents accept the situation and find new meanings in challenging circumstances should be provided.

\section{Conflict of interest}

No existing or potential conflict of interest relevant to this article was reported.

\section{Acknowledgements}

We would like to express our gratitude to the adolescents and their parents for consenting to participate in the study.

\section{REFERENCES}

1. Phipps S, Klosky JL, Long A, Hudson MM, Huang Q, Zhang H, et al. Posttraumatic stress and psychological growth in children with cancer: Has the traumatic impact of cancer been overestimated? Journal of Clinical Oncology. 2014;32(7):641-646. https://doi.org/10.1200/JCO.2013.49.8212

2. Barakat LP, Alderfer MA, Kazak AE. Posttraumatic growth in adolescent survivors of cancer and their mothers and fathers. Journal of Pediatric Psychology. 2006;31(4):413-419. https://doi.org/10.1093/jpepsy/jsj058

3. Tedeschi RG, Calhoun LG. Posttraumatic growth: Conceptual foundations and empirical evidence. Psychological Inquiry. 2004; 15(1):1-18.

4. Calhoun LG, Tedeschi RG. The foundations of posttraumatic growth: An expanded framework. In: Calhoun LG, Tedeschi RG, editors. Handbook of posttraumatic growth: Research and practice. 1st ed. Mahwah, New Jersey: Lawrence Erlbaum Associates; 2006. p. 1-23.

5. Haddy TB, Mosher RB, Reaman GH. Late effects in long-term survivors after treatment for childhood acute leukemia. Clinical Pediatrics. 2009;48(6):601-608.

https://doi.org/10.1177/0009922809332680

6. Hockenberry MJ, Wilson D, Rodgers CC. Wong's essentials of pe- 
diatric nursing. 10th ed. St. Louis, Misouri: Elsvier; 2017. p. 429500 .

7. Son SY. Illness experience of adolescents with hematologic malignancies. Journal of Korean Academy of Nursing. 2011;41(5):603612. https://doi.org/10.4040/jkan.2011.41.5.603

8. Hullmann SE, Fedele DA, Molzon ES, Mayes S, Mullins LL. Posttraumatic growth and hope in parents of children with cancer. Journal of Psychosocial Oncology. 2014;32(6):696-707. https://doi.org/10.1080/07347332.2014.955241

9. Zebrack B, Kwak M, Salsman J, Cousino M, Meeske K, Aguilar C, et al. The relationship between posttraumatic stress and posttraumatic growth among adolescent and young adult(AYA) cancer patients. Psycho-Oncology. 2015;24(2):162-168. https://doi.org/10.1002/pon.3585

10. Phipps S, Long A, Hudson M, Rai SN. Symptoms of post-traumatic stress in children with cancer and their parents: Effects of informant and time from diagnosis. Pediatric Blood and Cancer. 2005; 45(7):952-959. https://doi.org/10.1002/pbc.20373

11. Yonemoto T, Kamibeppu K, Ishii T, Iwata S, Tatezaki S. Posttraumatic stress symptom (PTSS) and posttraumatic growth (PTG) in parents of childhood, adolescent and young adult patients with high-grade osteosarcoma. International Journal of Clinical Oncology. 2012;17(3):272-275. https:// doi.org/10.1007/s10147-011-0286-3

12. Faul F, Erdfelder E, Lang AG, Buchner A. G*Power 3: A flexible statistical power analysis program for the social, behavioral, and biomedical sciences. Behavior Research Methods. 2007;39(2):175191. https://doi.org/10.3758/BF03193146

13. Tedeschi RG, Calhoun LG. The Posttraumatic growth inventory: Measuring the positive legacy of trauma. Journal of Traumatic Stress. 1996;9(3):455-471. https://doi.org/10.1002/jts.2490090305

14. Song SH, Lee HS, Park JH, Kim KH. Validity and reliability of the Korean version of the posttraumatic growth inventory. The Korean Journal of Health Psychology. 2009;14(1):193-214. https://doi.org/10.17315/kjhp.2009.14.1.012

15. Jo SM. The causal relationship of cognitive factors, social support and resilience on youth's posttraumatic growth [master's thesis]. Pusan: Pusan National University; 2012. p. 1-85.

16. Cann A, Calhoun LG, Tedeschi RG, Kilmer RP, Gil-Rivas V, Vishnevsky T, et al. The Core Beliefs Inventory: A brief measure of disruption in the assumptive world. Anxiety, Stress, and Coping. 2010;23(1):19-34. https://doi.org/10.1080/10615800802573013

17. Weiss DS, Marmar CR. The Impact of Event Scale-Revised. In:
Wilson JP, Keane TM, editors. Assessing psychological trauma and PTSD. 1st ed. New York: The Guilford Press; 1997. p. 399-411.

18. Lim HK, Woo JM, Kim TS, Kim TH, Choi KS, Chung SK, et al. Reliability and validity of the Korean version of the Impact of Event Scale-Revised. Comprehensive Psychiatry. 2009;50(4):385-390. https://doi.org/10.1016/j.comppsych.2008.09.011

19. Ahn HN, Joo HS, Min JW, Sim KS. Validation of the event related rumination inventory in a Korean population. Cognitive Behavior Therapy in Korea. 2013;13(1):149-172.

20. Cann A, Calhoun LG, Tedeschi RG, Triplett KN, Vishnevsky T, Lindstrom $\mathrm{CM}$. Assessing posttraumatic cognitive processes: The event related rumination inventory. Anxiety, Stress, and Coping. 2011;24(2):137-156. https://doi.org/10.1080/10615806.2010.529901

21. Nenova M, DuHamel K, Zemon V, Rini C, Redd WH. Posttraumatic growth, social support, and social constraint in hematopoietic stem cell transplant survivors. Psycho-Oncology. 2013;22(1): 195-202. https://doi.org/10.1002/pon.2073

22. Hungerbuehler I, Vollrath ME, Landolt MA. Posttraumatic growth in mothers and fathers of children with severe illnesses. Journal of Health Psychology. 2011;16(8):1259-1267. https://doi.org/10.1177/1359105311405872

23. Ministry of Health and Welfare, Korea Central Cancer Registry, National Cancer Center. Annual report of cancer statistics in Korea in 2013. Annual Report. Seoul: 2015 December. Report No.: 111352000-000145-10.

24. Pemberger S, Jagsch R, Frey E, Felder-Puig R, Gadner H, KryspinExner I, et al. Quality of life in long-term childhood cancer survivors and the relation of late effects and subjective well-being. Supportive Care in Cancer. 2005;13(1):49-56. https://doi.org/10.1007/s00520-004-0724-0

25. Nakayama N, Mori N, Ishimaru S, Ohyama W, Yuza Y, Kaneko T, et al. Factors associated with posttraumatic growth among parents of children with cancer. Psycho-Oncology. 2017;26(9):1369-1375. https://doi.org/10.1002/pon.4307

26. Tamres LK, Janicki D, Helgeson VS. Sex differences in coping behavior: A meta-analytic review and an examination of relative coping. Personality and Social Psychology Review. 2002;6(1):2-30. https://doi.org/10.1207/S15327957PSPR0601_1

27. Yonemoto T, Kamibeppu K, Ishii T, Iwata S, Hagiwara Y, Tatezaki SI. Psychosocial outcomes in long-term survivors of high-grade osteosarcoma: A Japanese single-center experience. Anticancer Research. 2009;29(10):4287-4290. 